\title{
161. Cranioplasty for Depressed Skull Fructure employing a Dental Drill
}

\author{
Masaru Mine, Mitsuo Toyama, Seishi Fukuma and Shigenobu Taketomo \\ 1st Dept. of Surgery, Kyoto Prefectural University of Medicine
}

A classical method to elevate a depressed skull fracture is to make a small burr hole at one edge of the depression for insinuation of flat periosteal instrument. But difficulties are encountered occasionally when the infolding fragments are firmly wedged to defy the crowbar action of the instrument, and underlying dural perforation is sometimes overlooked. In 1961, Masaru Mine, devised a simple technique employing a dental drill to elevate the depressed skull fracture. This method has been employed for 9 cases with good results. A Y-shaped trench is made along the slit in the center of the depression and fragments are elevated by crowbar action of periosteal instrument through the trench. As a rule, the underlying dura mater is torn, if involved, at the center whereupon the first trench is placed to provide inspection of perforation of the dura or subdural pathology if any.

\section{On the Repair of Dural Defect by use of Titanium-screen}

\author{
Nobuo HusHIKI \\ Shinko Clinic
}

Titanium was employed as a non-alloyed material in orthopedic treatment of fracture by Bothe et al. in 1940, and scanty responsiveness of this metal to host was clarified.

Titanium-screen- $0.2 \mathrm{~mm}$ thick, $1 \mathrm{~cm}$ square plate having gauzelike flexibility induced mainly from many small pores produced by photoetching method-was investigated, whether it may be useful as a dura substitute, experimentally using grownup dogs.

In these experiments:

1) Spectrographically, little dissolution of this metal to neighbouring tissues was clarified.

2) Histologically, titanium-screen was wrapped perfectly in cicatricial tissue in 4,8 and 12 weeks after insertion respectively. And the surface adjacent to brain was covered with layers of dural epithels.

These histological results were better than that of fascial autograft and tetron-mesh which were investigated simultaneously in each week. 
Finally, it may be said that these results indicate clinical superiority c titanium-screen as a dura substitute.

\title{
163. Mechanism and Dynamics of Closed Head Injuries (Preliminary Report)
}

Keiji Sano, Norio Nakamura, Kimiyoshi Hirakawa, Katsuoki AKashi, Hideaki Masuzawa, Keizo Hashizume, Shogi Ryn, Shigeru Kobayashi, Shuji Kamano and Hiroaki Sekino

Dept. of Neurosurgery, Univ. of Tokyo

(see the text)

\section{Studies on the Diagnosis and the Genetic Factors of Contre Coup Injury}

\author{
Haruyuki Kanaya, Ken-ichi Ohsawa, Danya Hirooka, Yoshihiko Kita, \\ Osamu KumagaI and Kyoji MiYamoto \\ 2nd Surgical Clinic, Iwate Medical University School of Medicine
}

Eighteen cases of cerebral lesion of contre coup type were experienced fo the past seven years among 22 autopsied cases of closed severe head injury. Thi disease has rather severe clinical symptoms while it is hard to recognize an! objective symptoms especially the course of impact, which has a diagnostic im portance.

That all of those 18 autopsied cases disclosed the fracture on the side opposit to cerebral lesion gives suggestion as to the course of impact. Therefore, fractur found by roentgenography should be valued on diagnosing contre coup injury.

Autopsy resulted lesion the most frequently at the frontal and temporal lobe $10(56 \%)$, following the temporal lobe, $5(28 \%)$ and the frontal lobe, $3(16 \%)$ and fracture at the occipital, $8(44 \%)$, the occipital and temporal, $4(22 \%)$ the temporal, $4(22 \%)$, and the frontal, $2(11 \%)$. Most contre coup injurie were found when fracture was at the occipital. Also, fracture of this part is mos likely accompany fracture of the base of the skull, so the roentgenography of th base of the skull is a necessary examination.

In conclusion, although autopsy does not totally clearify the genetic factor of contre coup injury, experimentally, intracranial negative pressure at the tim of fracture could be considered one of the influential factors. 